\title{
Study on the Intervention of Depression in Dying Patients from the Perspective of Music Psychology
}

\author{
Ning Qu \\ College of Humanities, Jiangxi University of Traditional Chinese Medicine, JiangXi Nanchang, \\ 330000, China
}

Keywords: Music, Terminal stage patients, Depression problems.

\begin{abstract}
Objective: To help dying patients to enhance self-concept, to promote relaxation, relieve stress, reduce depression. Methods: On the one hand, the subjects were interviewed to understand the basic status of dying patients. On the other hand, the depressive state of 30 patients with death was evaluated by SDS scale. The patients were randomly divided into experimental group, control group and experimental group The methods and methods of treatment were used to treat the depressive state of the patients with death. The results of SDS before and after the experiment were analyzed statistically. Results: The depressive state of the patients treated in the treatment group was significantly lower than that in the control group $(\mathrm{P}<0.05)$. Conclusion: The use of various forms and functions of music can effectively improve the state of depression in dying patients is a better psychological crisis intervention.
\end{abstract}

\section{Introduction}

Life is the most precious thing in life, from birth to death, respect for life and care is a manifestation of good human nature. The traditional concept of doctors, dying patients and their families one-sided pursuit of "endless life, treatment more than", and finally both sides have to face "suffered toss, wealth and two empty" helpless ending. Although the spirit of life and active treatment worthy of advocacy, but in the life of the end of the period, dying patients not only have to endure physical illness and treatment of side effects, but also bear the fear of death, family guilt and the economy Stress and other spiritual aspects of the burden of its quality of life has been greatly affected. Hospice care is a special kind of palliative care service, not to extend the survival time for the purpose, and to improve the treatment of patients hopeless life quality of life for the purpose. In 1988, China began to establish a hospice care service for patients, since then, hospice care services have been carried out in our country. In 2000, the study of hospice research literature showed a slow development trend, many institutions for the field of research in the wait and see stage. By 2006, there has been a rapid growth in this field, and hospice care has received increasing attention from all walks of life. At present, China's implementation of hospice care for patients mainly in psychological intervention, nutrition, comfort and so on, mainly to language communication, means a single, the form is not flexible and diverse. Studies have shown that when some emotions are difficult to express through the language, the music can be effectively used as a means of communication and emotional establishment of the bridge to help dying patients and their families catharsis bad mood, the establishment of good relations. The hospice care of music as a means of communication and way is slightly mentioned in the domestic research literature, but there is no special study of institutions and literature.

This research is based on the humanities and social science projects of the Ministry of Education to build up, from the perspective of music psychology to explore the music in the hospice of the mechanism of research, in order to better improve the hospice music intervention mechanism. 


\section{Patient basic information}

The patients were randomly divided into experimental group and control group. There was no significant difference in age, education and gender between the two groups $(\mathrm{p}>0.05)$. There were no significant differences between the two groups in the age group, Indicating that the two groups of patients with end of the comparable, see Table 1.

Table 1. Comparison of basic information between two groups of dying patients $\left(\bar{X}_{ \pm} s\right)$

\begin{tabular}{cccccc}
\hline & & $\overline{\mathrm{x}} \pm \mathrm{s}$ & $\mathrm{T}$ & 2 & $\mathrm{p}$ \\
\hline \multirow{2}{*}{ Age } & Control group & $64.27 \pm 7.778$ & -0.58 & & 0.56 \\
& Intervention group & $65.67 \pm 5.052$ & 5 & & 3 \\
\hline \multirow{2}{*}{ Education } & Control group & $2.67 \pm 0.900$ & & 1.90 & 0.59 \\
$\mathrm{n}$ & Intervention group & $2.40 \pm 0.986$ & & 5 & 2 \\
\cline { 2 - 4 } Sex & Control group & $1.40 \pm 0.507$ & & 1.20 & 0.27 \\
\cline { 2 - 3 } & Intervention group & $1.60 \pm 0.507$ & & 0 & 3 \\
\hline
\end{tabular}

There was no significant difference between the two groups ( $>0.05)$. There was no significant difference between the two groups $(\mathrm{P}>0.05)$. There was no significant difference between the two groups ( $\mathrm{p}>0.05)$.

Exclusion criteria are: (1) do not want to participate in music therapy activities or do not want to accept random distribution; (2) the existence of mental illness; (3) there are serious hearing disorders, speech disorders and cognitive disorders; (4) brain degeneration.

\section{Experimental Study}

\section{Research process}

The study was divided into three procedures. First, all dorsal patients were interviewed and evaluated by SDS scale. Secondly, the patients were randomly divided into two groups. According to the results of the previous evaluation, the experimental group did not carry out the intervention of the music therapy in the control group. Music therapy interventions twice a week, each time about 40-60 minutes depending on the state of the dying patient. Finally, two sets of dying patients were reassessed after 16 episodes of music.

\section{Research tools}

\section{Psychology tools}

According to the study, the questionnaire is divided into two parts, some of which are the basic situation of the dying patients, including age, sex, hobbies, music background and other related issues; the other part is to investigate the mental symptoms and physical symptoms of the dying patients, including mood , Energy, memory, sleep, suicidal tendencies and so on.

SDS Depression Rating Scale is a self-rated rating of 4 ratings, with a total of 20 entries. Easy to use, easy to operate is the largest feature of this scale, can visually reflect the subjective feelings of depressed patients reflected. The scope of application of SDS is mainly adults who show depressive symptoms. This scale mainly responds to four groups of specific symptoms of depression: psychotic - emotional symptoms, somatic disorders, psychomotor disorders, and psychological disorders of depression. The lower the score, the better the state of the patient is, and vice versa.

Therapeutic instruments

Music and sound equipment, piano, Ukulele, xylophone, bell piano, various types of drums

\section{Research content}

The establishment of treatment relationships. The patient's sense of trust and intimacy of the music therapist is an important factor in the success of the treatment. The establishment of the treatment of 
the dynamic relationship in the course of the activity is the basis for achieving results. Only on the basis of good relations can the patient be positively motivated and willing to Play the potential and power, this stage will run through the entire treatment period. At this stage the therapist can communicate with the dying patient through music singing, including daily greetings, conversations, and ends. In this process the therapist needs a compassionate ability to experience the patient's inner world.

The use of music psychological emotions, cognitive and social functions, to deal with dying patients emotional, cognitive, social and other aspects of the problem, and ultimately ease the dying patients with depression. The main techniques used are: music progressive relaxation technology, providing a variety of suitable for the relaxation of the type of music for the subjects to choose, or by the subjects to make him feel relaxed music, by the therapist to assess whether to meet the requirements of relaxation music. The music and relaxation guide language combination, so that the subjects to relax the muscles, breathing steady, heart rate, ease depression, improve sleep conditions. Song singing, through the therapist and the patient singing their special significance to the song, to help the dying patient due to the environment caused by the boring sense of the transfer of pain or adverse symptoms of attention. Positive resources to imagine, to help patients find positive experiences in the past life, to strengthen the positive experience, thereby reducing the negative impact of negative emotions. Music life review, let the patients from their childhood to the important life of the important period or the importance of the song or music in order to arrange a good order to form a system of personal music history, and share every period of each The first music, to help patients remember all the unforgettable stories in this life. Music memories, to arouse the positive memories of life, to strengthen the value of life affirmed. Song discussion, with the theme of the song discussion, not only in the psychological level to support the role of patients, but also can help patients to re-cognition of death, reduce the fear of death, music creation, therapist to help create Will be the patient, for the song re-word or innovative songs for the family left a personal creative audio and video information, to maintain the emotional connection between patients and their families. In addition to the above technology, there are a lot of technology can use the various elements of music or activities to help the dying patients to change the cognitive, ease the pain eventually ease the depression, improve the quality of life.

\section{Statistical processing}

Statistical analysis SPSS17.0 statistical software was used to analyze the data of each group, and the control group and the experimental group were analyzed by $\mathrm{T}$ test.

\section{Results}

\section{Interview}

Through interviews and questionnaires learned that patients have a certain preference for music, the age or life in the important stage of the song has a preference. In addition from the physiological point of view, part of the dying patients due to a large number of chemotherapy and drug side effects, resulting in no appetite, poor quality of sleep and with a serious sense of fatigue, easily lead to depression. From a psychological point of view, most of the dying patients due to the wrong perception of death and have a serious sense of fear, a lot of medical expenses will cause the family's guilt, slow thinking, self-enclosed, severe depression. 


\section{SDS before and after comparison}

Table 2. SDS control group, the experimental group before and after the test with the sample T test

\begin{tabular}{cccccc}
\hline Group & Stage & Number of cases & $\bar{x} \pm s$ & $\mathrm{~T}$ & $\mathrm{P}$ \\
\hline \multirow{2}{*}{ Control group } & Before intervention & 15 & $47.80 \pm 3.53$ & \multirow{2}{*}{-0.16} & \multirow{2}{*}{0.88} \\
\cline { 2 - 5 } & After intervention & 15 & $47.87 \pm 3.29$ & & \\
\cline { 1 - 5 } Intervention group & Before intervention & 15 & $48.73 \pm 3.67$ & 16.3 & \\
\cline { 2 - 5 } & After intervention & 15 & $30.13 \pm 4.82$ & 6 & $0.00^{* *}$ \\
\hline
\end{tabular}

Note: $* * \mathrm{P}<0.01$

The results showed that there was no significant difference between the control group and the control group before and after the intervention, and there was significant difference $(P<0.01)$ in the intervention group before and after the intervention, indicating that the intervention had a good effect on reducing the depressive mood of the subjects.

\section{Discussion and conclusions}

In foreign countries, a large number of data show that when the drug treatment has been ineffective for dying patients, music as an auxiliary means can effectively meet the dying patient mood and mental needs, relieve symptoms and help dying patients improve the quality of life. In the developed country's hospice care institutions, such as the United States of Montana, Missouri, St. Petek Hospital, Montenegro, the music therapist Terence has developed a "peace plan", she called her work for "music death", According to the plan in the nursing center to develop hospice care of the basic items. In foreign hospice care institutions, music has become the most popular adjuvant therapy in hospice care services, to help dying patients to reduce fear and anxiety, enhance self-concept, promote relaxation, ease the symptoms of insomnia, reduce loneliness. Dying patients participate in music activities at the same time, not only to enhance emotional vitality and emotional support, but also help dying patients to reduce pain and drug treatment and other side effects. The role of music in hospice care is a powerful tool to improve the quality of life, fully embodies the needs of humanistic care and the unity of quality of life and value.

This will not only help to study the special group of patients with hospice care, to promote further research and research in this field, to strengthen the hospice service effectiveness, to promote the development of the discipline, but also the comprehensive development of music in the field of treatment and the formation of a relative system Theory to provide effective theoretical reference and support. The thesis will form a theoretical basis for the future research of music therapy in this field, and also provide an effective basis for the clinical treatment of music therapy in China, so it has important theoretical and practical value.

\section{Acknowledgments}

This article is the result of the study of the hospice care in the view of the humanities and social sciences of Jiangxi Province (JC162002) ; the result of the study of the music intervention of the psychological crisis of the dying family members of the Jiangxi province's cultural and artistic science project(YG2016117). This part of the paper has been the focus of the State Administration of Traditional Chinese Medicine Cultivate the discipline "Chinese medicine psychology", Jiangxi province culture and art science research base "Gan Po Chinese medicine culture psychology research center" funding.

\section{References}

[1] S.Munro \& B.Mount. Music therapy in palliative care. Canadian Medical Association Journal, 1978,119(9): 1029-1034. 
[2] Qu Ning.Research on the Social Investigation of Music Therapy in China's Hospice Area. China Public Health, 2016.6.

[3] Li Yiting, Li Wei. Hospice care. Beijing: China Science and Technology Press. 2003.

[4] Qu Ning, Duan Senhai.Research on the Current Situation of Music Therapy in China's Hospice. Science and technology winds, 2015.09.

[5] Qu Ning, Zeng Qingxia, Ren Yuan. Clinical Study on the Effect of Group Music on Relieving Psychological Pain in Late - stage Cancer Patients. Daily health, 2016,02. 